\title{
X008
}

\section{Estimating the Location of Scatterers by Seismic Interferometry of Scattered Surface Waves}

\author{
U. Harmankaya* (Istanbul Technical University), A. Kaslilar (Istanbul \\ Technical University), J. Thorbecke (Delft University of Technology), K. \\ Wapenaar (Delft University of Technology) \& D. Draganov (Delft \\ University of Technology)
}

\section{SUMMARY}

In this study, non-physical (ghost) scattered surface waves are used to obtain the location of a near-surface scatterer. The ghost is obtained from application of seismic interferometry to only one source at the surface. Different locations for virtual sources are chosen and ghost scattered surface waves for each of these virtual-source locations are retrieved. The retrieved ghost traveltimes are inverted by solving the inverse problem to determine the location of the scatterer. It is seen that the location of the scatterer is reasonably well estimated. 


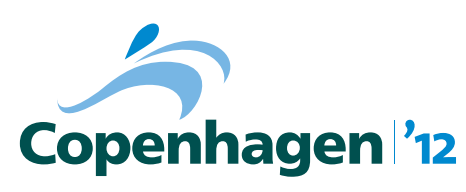

\section{Introduction}

Near-surface structures such as cavities, caves, tunnels, mineshafts, buried objects, archeological ruins, water reservoirs and similar, cause scattered surface waves. These near-surface scatterers may pose risk during and after the construction of buildings, transportation ways (roads, highways, railways) or power plants (wind, solar, etc) which are spread to wide areas. These scatterers can be affected by the changes in the hydraulic regime, earthquakes and change of the loading on the soil and may pose hazards. Therefore, the detection and monitoring of this type of weak zones is important to mitigate environmental and geohazards.

Several authors used scattered surface waves for imaging cavities, buried objects, or shallow water reservoirs (Snieder, 1987; Herman et al, 2000; Campman and Riyanti, 2007; Kaslilar and Herman, 2006; Kaslilar, 2007). The scattered surface waves are studied in detail in terms of seismic interferometry by Halliday and Curtis (2009).

In this study the correlation-type interferometric estimate of the ghost scattered surface waves is used for obtaining the location of a near-surface scatterer. The scattered wavefield is modeled using the integral representation of the scattered wavefield given in Kaslilar (2007). For selected virtual-source locations, the interferometric estimates of the ghost scattered wavefields are obtained. The traveltimes of the ghosts are picked and inverted by using the theoretical ghost traveltimes. The end results are the horizontal and vertical locations of the scatterer.

It is anticipated that the introduced method will be more effective than other methods when lateral changes of the medium properties, such as velocity gradient or random inhomogeneties, are present. As seismic interferometry effectively redatums sources (or receivers) from places away from the scatterers to the target area, the unwanted extra effects, due to propagation from sources through the laterally changing medium and/or scatterers to the receivers close to the target area, are eliminated. Using the interferometric traveltimes of the ghosts the scatterers can be located. The method can also reduce the calculation times for waveform inversion studies. Although this study is initiated at geotechnical scale, the suggested method is not restricted to geotechnical studies. It can also be used in exploration and global seismology for detecting the near-surface scatterers.

\section{Calculation of the Interferometric Ghost of the Scattered Surface Waves}

To calculate the scattered wavefield, the computationally efficient method developed by Kaslilar (2007) is used. In this method three dimensional propagation and scattering of elastic waves are considered in an isotropic, laterally homogeneous embedding in which bounded objects with contrasting density are present. Since in the method the total wavefield is obtained as the sum of the incident wavefield and the scattered wavefield $u_{i}=u_{i}^{i n c}+u_{i}^{s c}$, only the scattered part of the wavefield is used in the modeling and in retrieving of the ghost scattered surface waves. Having the chance of using only the scattered part of the wavefield is important for testing the success of the suggested method. With this opportunity, the direct Rayleigh waves, which dominate the interferometric image, are not present in the wavefield and the traveltimes of the interferometric estimate corresponding to ghost scattered surface waves are easily selected.

Considering the Born approximation, the scattered wavefiled $u_{i}^{s c}$ is calculated by using the following domain type integral representation,

$$
u_{i}^{s c}\left(x^{r}, \omega, x^{s}\right)=\omega^{2} S(\omega) \int_{D} \sigma\left(x^{\prime}\right) u_{i j}^{G}\left(x^{r}, \omega, x^{\prime}\right) u_{j 3}^{G}\left(x^{\prime}, \omega, x^{s}\right) d V\left(x^{\prime}\right),
$$

where the source the receiver and scatterer locations, the angular frequency and the source waveform are denoted by $x^{s}, x^{r}, x^{\prime}, \omega$ and $S(\omega)$, respectively. In Eq. (1), $\sigma$ is the density contrast value, being the difference between the scatterer density $\rho_{s c}$ and the background density $\rho_{0} ; u_{j 3}^{G}$ is the Green's displacement tensor of the incident wavefield from the source to the scatterer generated by a 


\section{Copenhagen '12}

vertical point force, and $u_{i j}^{G}$ is the Green's displacement tensor of the wavefield from the scatterer to the receiver.

For the modelling the parameters from the ultrasonic laboratory experimental set-up described in Kaslilar (2007) are used, since a laboratory experiment is planned for future studies. The geometry and the medium parameters of the model are given in Figure 1.

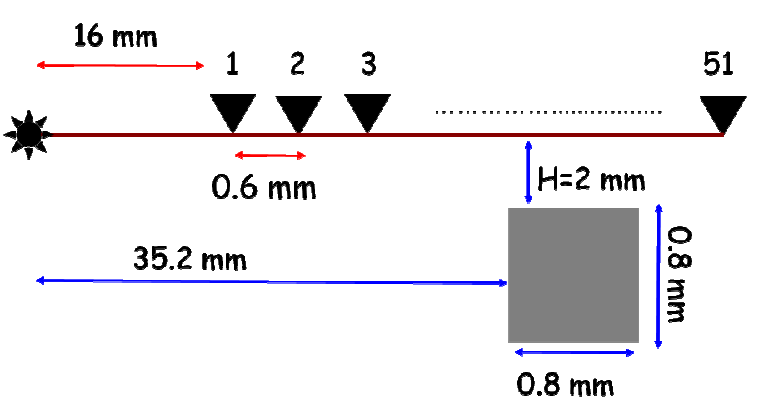

\begin{tabular}{|l|c|c|}
\hline & $\begin{array}{c}\text { Background } \\
\text { (aluminum) }\end{array}$ & $\begin{array}{c}\text { Scatterer } \\
\text { (epoxy) }\end{array}$ \\
\hline$\rho\left(\mathrm{kg} / \mathrm{m}^{3}\right)$ & 2700 & 7000 \\
\hline $\mathrm{V}_{\mathrm{p}}(\mathrm{m} / \mathrm{s})$ & 5700 & 5700 \\
\hline $\mathrm{V}_{\mathrm{s}}(\mathrm{m} / \mathrm{s})$ & 3080 & 3080 \\
\hline
\end{tabular}

Figure 1 Schematic view of the scale model (left):the source (star), receivers (triangles) and scatterer (grey square). The modelling parameters for the background medium and the scatterer are given in the table (right).

The dominant wavelength of Rayleigh wave is $\lambda_{d} \cong 2.85 \mathrm{~mm}$ since the dominant frequency is $1 \mathrm{MHz}$. A receiver line with 51 receivers having a sampling of $0.20 \lambda_{d}(0.6 \mathrm{~mm})$ is placed above a scatterer of approximately $0.30 \lambda_{d}$ in size. Using the configuration and the medium parameters the scattered wavefield shown in Figure 2a is calculated. The scattered P and Rayleigh waves can be seen in Figure 2a. Seismic interferometry is applied to these scattered waves by using only one source and by crosscorrelating the reference trace $d^{V S}$ (the trace at the virtual source position) with the rest of the traces, $d^{i}$, which are present on the seismic record. This relation is

$$
C_{d^{i} d^{V S}}(\tau)=\sum_{t} d_{t}^{i} d_{t+\tau}^{v S} \text {. }
$$

Note that as the source is at the surface while the scatterer is at depth, the source is not at the stationary point for retrieving a physical scattered surface waves. Application of Eq. (2) will eliminate the common travel path from the source to the scatterer and will result in the retrieval of a ghost scattered surface wave. As the modeled buried object is a scatterer, it scatters the illumination wavefield in the same way irrespective of the position of the surface source. For this reason, the retrieved ghost scattered surface waves will be the same for any position of the surface source, except for the case when losses are present in the medium. In the latter case, the only change in the retrieved ghost will be in its dominant frequency. In Figure $2 b-d$ the retrieved ghost scattered surface waves for virtual-source locations at receivers 1, 26 and 40 are given respectively. It can be seen that the scattered fields are the same, but for displacement along the time axis. This displacement depends on the distance from the virtual source to the scatterer only. Change in the lateral direction of the medium parameters to the left and to the right of the receiver array will not affect the retrieved ghost traveltimes. The ghost traveltimes are picked from the maximum amplitude of the retrieved ghost scattered surface waves since in the modeling a zero-phase band-pass signal is used as a source wavelet. The picked traveltimes are shown by the red curves on Figure $2 b-d$. In the next section these traveltimes are used for obtaining the location of the scatterer.

\section{Estimation of the Location of the Scatterer}

To estimate the location of the scatterer, the following theoretical ghost travel time relation is used,

$$
\tau=\frac{1}{V_{R}}\left\{\left[\left(x_{V S}-x_{S C}\right)^{2}+\left(z_{V S}-z_{S C}\right)^{2}\right]^{1 / 2}-\left[\left(x_{i}-x_{S C}\right)^{2}+\left(z_{i}-z_{S C}\right)^{2}\right]^{1 / 2}\right\} .
$$




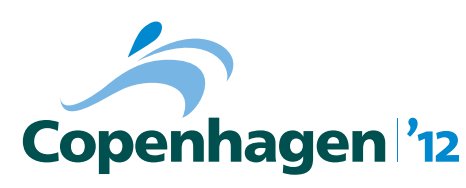

The relation gives the retrieved ghost traveltimes between the virtual source, the scatterer and the receivers. In the equation, $V_{R}$ is the Rayleigh wave velocity, $i$ is the index for the receiver numbers, $V S$ and $S C$ denote the virtual source and the scatterer, respectively, while $x$ and $z$ are the location coordinates in horizontal and vertical direction, respectively.
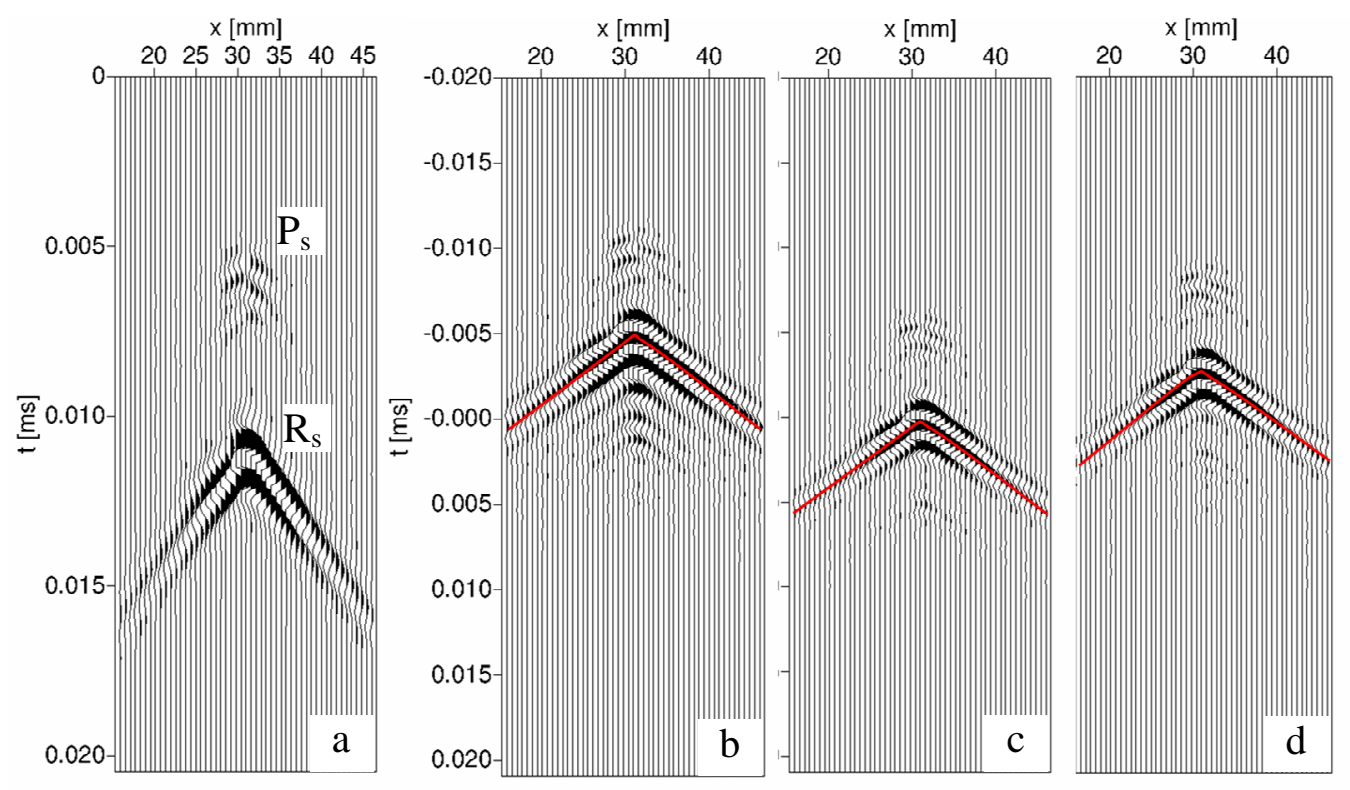

Figure 2 (a) The modeled scattered wavefield, $P_{s}$ and $R_{s}$ denote the scattered $P$ and Rayleigh waves, (b), (c) and (d): Ghost scattered surface waves retrieved by applying seismic interferometry to (a) for virtual source locations 1, 26 and 40, respectively. Figures are plotted by Seismic Un*x (Cohen and Stockwell, 2000)

To find the location of the scatterer, the traveltime relation (Eq.3) and the traveltimes obtained for each virtual source location (red curves in Figure $2 b$ to $d$ ) are used in the inversion. The nonlinear problem is solved iteratively. The system of equations for the forward problem is denoted as $\Delta \mathbf{d}=\mathbf{G} \Delta \mathbf{m}$. In this relation, the difference between the observed $\tau_{o b s}$ (retrieved), and the calculated $\tau=\tau_{\text {calc }}$ (Eq.3) ghost scattered data is denoted by $\Delta \mathbf{d}=\tau_{\text {obs }}-\tau_{\text {calc }}$, the unknown model parameters - the horizontal $x_{s c}$ and vertical $z_{s c}$ locations of the scatterer - are denoted by the vector $\Delta \mathbf{m}$, while the Jacobian matrix is represented by $\mathbf{G}$. The damped least-squares solution of the inverse problem is given in terms of Singular Value Decomposition (SVD) as,

$$
\Delta \mathbf{m}=\mathbf{V} \boldsymbol{\Lambda}\left(\boldsymbol{\Lambda}^{2}+\beta^{2} \mathbf{I}\right)^{-1} \mathbf{U}^{T} \Delta \mathbf{d},
$$

where $\mathbf{V}, \boldsymbol{\Lambda}, \mathbf{U}, \mathbf{I}$ and $\beta$ are the model-space eigenvectors, the diagonal matrix containing the eigenvalues, the data-space eigenvectors, the identity matrix and the damping parameter, respectively. Considering Eq. (4) the inverse problem is solved to find the location of the scatterer. The best fit between the observed and calculated traveltimes of the ghost scattered surface waves for virtual sources 1, 26 and 40 are given in Figure 3a and the estimated model parameters are given in Figure $3 b$.

It can be seen that there is a good agreement between the observed and the calculated traveltimes of the ghost scattered surface waves. The initial and the updated model parameters for each iteration are given in Figure 3b. After five iterations, the model parameters, the horizontal and vertical location of the scatterer, get closer to the actual values. It is observed that the location of the scatterer is reasonably well estimated. It should be noted that the starting point of the coordinate system is arbitrarily chosen and it starts at the position of the actual source. 
Copenhagen '12

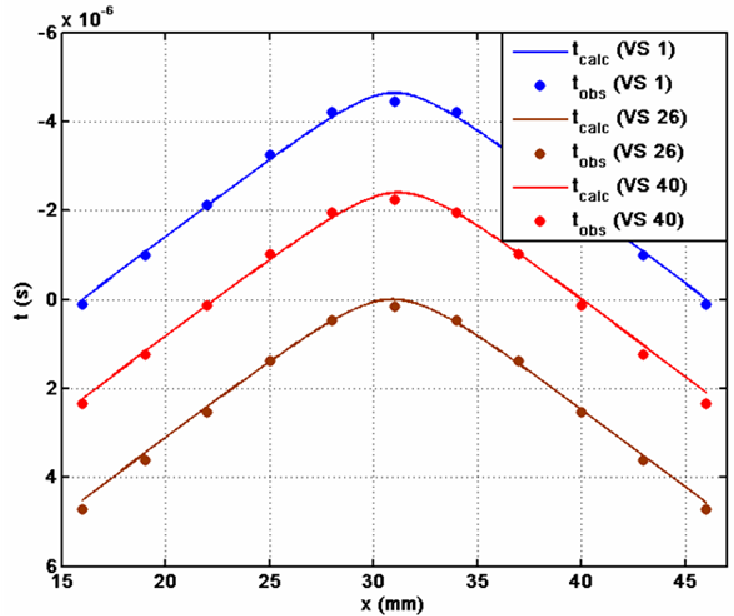

(a)
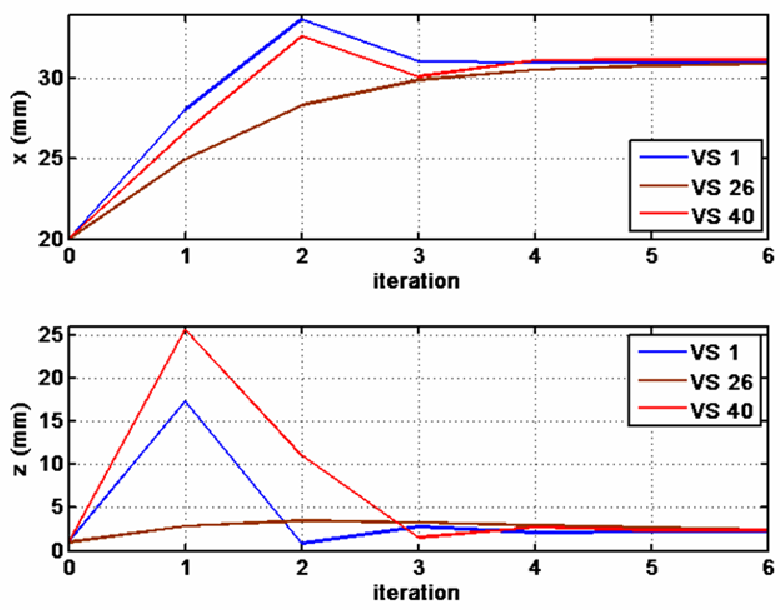

(b)

Figure 3 (a) Observed and calculated travel times, (b) estimated horizontal and vertical locations of the scatterer for the virtual sources 1(blue), 26(brown) and 40 (red).

\section{Conclusion}

A method for obtaining the location of a near-surface scatterer is proposed by using traveltimes of non-physical (ghost) scattered surface waves retrieved from seismic interferometry. The ghost scattered surface waves are obtained by cross-correlating the recorded scattered surface waves originating from only one source at the surface. The traveltimes of the ghost scattered surface waves are used in an inversion to find the location of the scatterer. The depth and the horizontal position of the scatterer is obtained for different virtual-source locations.

Advantage of the proposed method is that the unwanted travel paths between the source and the receiver array are eliminated. These travel paths can traverse a complicated medium. Due to elimination of these paths, the calculation times for waveform inversion studies can be reasonably reduced. Also when lateral changes of the medium properties are present, these path effects can be eliminated by interferometry and locations closer to the target can be considered for estimation of the location of the scatterer. Currently more realistic numerical models are studied for the interferometric estimates of the ghost scattered surface waves.

\section{Acknowledgements}

This work is supported by TUBITAK (The Scientific and Technological Research Council of Turkey) with the project 110Y250 titled "Detecting Near-surface Scatterers by Inverse Scattering and Seismic Interferometry of Scattered Surface Waves". We gratefully acknowledge this financial support.

\section{References}

Campman, X. and Riyanti, C. D., 2007, Non-linear inversion of scattered seismic surface waves, Geophys. J. Int. 171, 1118-1125.

Cohen, J. K. and Stockwell, Jr. J. W., (2000), CWP/SU: Seismic Un*x Release No. 34: an open source software package for seismic research and processing, Center for Wave Phenomena, Colorado School of Mines.

Herman, G.C., Milligan, P.A., Huggins, R.J. and Rector, J.W., [2000] Imaging shallow objects and heterogeneities with scattered guided waves. Geophysics, 65 (1), 247-252.

Kaslilar, A. and Herman, G.C., [2006] Imaging of scattered surface waves- Application to Ultrasonic Data. $68^{\text {th }}$ EAGE Conference \& Exhibition, Vienna, Austria, Extended Abstracts, H-009.

Kaslilar, A., [2007] Inverse scattering of surface waves: imaging of near-surface heterogeneities. Geophys. J. Int. 171, 352-367.

Snieder, R.K., [1987] Surface wave holography. In: Seismic tomography (ed. G.Nolet) D.Reidel Pub. Co., 323-337. 